\title{
Review
}

\section{Penaeidins, a family of antimicrobial peptides from penaeid shrimp (Crustacea, Decapoda)}

\author{
D. Destoumieux ${ }^{\mathrm{a}}$, M. Munoz ${ }^{\mathrm{a}}$, P. Bulet ${ }^{\mathrm{b}}$ and E. Bachère ${ }^{\mathrm{a}, *}$ \\ 'IFREMER/CNRS/Université Montpellier 2, UMR 219 'Défense et Résistance chez les Invertébrés Marins', \\ CC 80, Place Eugène Bataillon, 34095 Montpellier (France), e-mail: ebachere@ifremer.fr \\ bInstitut de Biologie Moléculaire et Cellulaire, UPR 9022, CNRS, 'Réponse Immunitaire et Développement \\ chez les Insectes', 15, rue René Descartes, 67084 Strasbourg Cedex (France)
}

Received 21 January 2000; received after revision 10 March 2000; accepted 10 March 2000

\begin{abstract}
The production of antimicrobial peptides represents a first-line host defense mechanism of innate immunity that is widespread in nature. Only recently such effectors were isolated in crustacean species, whereas numerous antimicrobial peptides have been characterized from other arthropods, both insects and chelicerates.
\end{abstract}

This review presents findings on a family of antimicrobial peptides, named penaeidins, isolated from the shrimp Penaeus vannamei. Their structure and antimicrobial properties as well as their immune function will be discussed through analyses of penaeidin gene expression and peptide distribution upon microbial challenge.

Key words. Crustacean; shrimp; innate immunity; antimicrobial peptides; structure; activity spectrum; blood cells; expression.

\section{Introduction}

The past few years brought significant advances in crustacean immunity, with a focus on antimicrobial peptides. These molecules, which are found in both the plant and animal (vertebrate and invertebrate) kingdoms, act as endogenous antibiotics and as such are considered to be a key element of innate immunity. A wide variety of antimicrobial peptides have been described to date. They are classified into three distinct groups based on amino acid sequences, secondary structures and functional similarities [1]. The first and largest group is composed of peptides stabilized by intramolecular disulfide bonds, and the two latter groups are linear peptides and polypeptides characterized by (i) $\alpha$-helical structures or (ii) a high content of proline

\footnotetext{
* Corresponding author
}

residues and/or a high percentage of glycine residues. Despite variations in size and structure, most of these molecules are amphiphilic, displaying both cationic and hydrophobic surfaces. Different mechanisms of action have been described. In most cases, antimicrobial peptides were shown to disrupt microbial membranes by a pore-forming action or by a detergent effect [2]. However, other peptides, such as the insect attacins, were found to block bacterial membrane biosynthesis, resulting in cell death [3].

Antimicrobial peptides are produced in phagocytic cells of vertebrates [4], invertebrates [5-7], and tunicates [8, 9], and are also found in various tissues, for instance in the mammalian intestinal epithelium [10] or in the insect fat body (a functional equivalent of the mammalian liver), which was found in this group to be the main site for antimicrobial peptide synthesis [11].

Depending on the species or tissues, their expression is known to follow different modes of regulation. In 
arthropods, peptide synthesis is rapidly induced in the insect fat body upon in vivo microbial challenge, but also aseptic injury [6]. Two pathways have been identified, namely Toll and Imd, which control the transcription of Drosophila melanogaster antifungal and antibacterial peptides, respectively [12]. In horseshoe crab hemocytes (invertebrate blood cells), antimicrobial peptides appear to be constitutively expressed, and further stored in cytoplasmic granules from which they are released, after contact with microbial endotoxins, by regulated exocytosis (degranulation) [13]. Both the insect and chelicerata systems are believed to end in an increased quantity of antimicrobial peptides in the plasma (cell-free hemolymph), resulting in a greater ability to fight microbial infection.

Until recently, the contribution of antimicrobial peptides to crustacean defense was only suspected. Bactericidal activities were observed in Homarus americanus plasma [14] and hepatopancreas [15], but were shown to be absent from the plasma of the crab Carcinus maenas, even after microbial challenge. From these observations, White et al. [16] have suggested that in crustaceans, elimination of invading organisms from the bloodstream is more likely to be mediated by hemocytes (through phagocytosis and further killing by cellular molecules) than by the presence of plasma-soluble antimicrobial factors.

Potent antibacterial activities in C. maenas hemocyte lysates that were not due to agglutinins or associated with the prophenoloxidase (proPO) activating system reinforced the idea of the existence of cellular antibacterial factors [17]. Similar observations were also made in the hemocytes of several other crustacean species [18], and over the last 4 years several peptidic factors displaying antimicrobial activities were isolated in crabs and shrimp. To date, antibacterial peptides and polypeptides were extracted from the hemocytes of the crabs C. maenas [19] and Callinectes sapidus [20], and two of them, a $6.5-\mathrm{kDa}$ peptide and the $3.7-\mathrm{kDa}$ callinectin, respectively, were partially characterized. In addition, a family of antimicrobial peptides displaying both antifungal and antibacterial activities was isolated from the hemolymph of the shrimp $P e$ naeus vannamei, and were named penaeidins [21]. The full characterization of penaeidins at the biochemical and nucleic acid level led to production of both recombinant peptides and specific molecular probes to study penaeidin antimicrobial activities and expression in shrimp subjected to microbial infection. Taken together, these new data provide clues to understanding the role of antimicrobial peptides in crustacean immune response.

\section{Penaeidin structure}

\section{Mature peptides}

Three penaeidins were purified from the hemolymph of the shrimp $P$. vannamei. The peptides were isolated in their active form $(5.48-6.62 \mathrm{kDa})$ in acid extracts from both plasma and hemocyte organelle-rich fractions of shrimps obtained from intensive Ecuadorian culture farms. The peptides were fully characterized at the amino acid (Pen-1, -2 and -3a) and nucleic acid (pen-2, $-3 a,-3 b$ and $-3 c$ ) levels, by a biochemical approach and complementary DNA (cDNA) cloning from a hemocyte library, respectively. Like most of the antimicrobial peptides hitherto described, penaeidins are highly cationic molecules with calculated isoelectric points ranging from 9.34 to 9.84 . The bioactive molecules show high levels of similarities in their amino acid sequences. They are composed of an $\mathrm{NH}_{2}$-terminal proline-rich region as observed for the other crustacean antimicrobial peptides $[19,20]$, followed by a $\mathrm{COOH}-$ terminal domain stabilized by three intramolecular disulfide crosslinks (fig. 1a). This unique structure led to the identification of a new family of antimicrobial peptides. Besides this chimeric-like overall structure, penaeidins display posttranslational modifications. In particular, Pen-2 and -3a are $\mathrm{COOH}$-terminally amidated in their mature form, but such a modification is still unknown in Pen-1, for which the cDNA sequence has not been established. Other marine invertebrate antimicrobial peptides, including the horseshoe crab tachyplesins [22] and polyphemusins [23], are also COOH-terminally amidated. In addition, Pen-3a is blocked at the $\mathrm{NH}_{2}$-terminus by a pyroglutamic acid which has also been reported in the insect hymenoptaecin [24] or some bovine $\beta$-defensins [25].

\section{Peptide processing}

The amino acid sequences deduced from the cDNA revealed that the penaeidins isolated from hemocytes are synthesized as precursor molecules consisting of a signal peptide (19-21 amino acids) immediately preceding the bioactive molecule [21]. Pen-2 and -3a signal peptides are fully identical with the exception of two additional amino acids (Glu-Ala) at the $\mathrm{COOH}$-terminal position in the Pen-2 signal sequence (fig. 1b). Since a potential cleavage site by signal peptidase is predicted at the $\mathrm{NH}_{2}$-terminal position of the glutamic acid residue [26], we can speculate that Pen-2 maturation involves an additional processing step compared with Pen-3a, possibly by a dipeptidyl amino peptidase (DPAP) (fig. 2). Such DPAPs are able to cleave at the COOH-terminal position of X-Pro and X-Ala dipeptides (as found in Pen-2), and their involvement in processing precursor molecules was previously shown 
for other antimicrobial peptides such as cecropins or mellitins [27]. As for Pen-2, cecropin and sarcotoxin IIA mature peptides are separated from their signal sequence by one or two X-Pro and X-Ala dipeptides [27, 28]. Similar maturation events, including elimination of signal sequence and cleavage by DPAPs, are believed to process the horseshoe crab tachyplesins [29], a family of antimicrobial peptides synthesized and stored within hemocytes after maturation, indicating that such a process may be at work in penaeidin processing in the shrimp blood cells.

\section{Recombinant penaeidins}

In order to produce large amounts of peptides for further structural studies or biological assays, penaeidins were synthesized in a heterologous expression system. Along with baculoviruses [30], yeasts are good candidates for heterologous expression of antimicrobial peptides containing disulfide-linked cysteine residues
[31-33]. Penaeidin-2 and -3a cDNAs were fused with the $\beta$-glucanase signal peptide and $\alpha$-mating factor pheromone propiece coding sequences, and this construct was used to tranfect Saccharomyces cerevisiae TGY [34]. In this system, mature penaeidins were secreted into the culture medium at concentrations of about $1 \mathrm{mg} / 1$. Since the antimicrobial activities of the recombinant penaeidins were almost indistinguishable from those of the native molecules, they were most likely correctly folded in yeast.

Further characterizations showed that recombinant penaeidins differ from the native molecules by the presence of an additional glycine residue at the $\mathrm{COOH}$-terminus, instead of the naturally occurring $\alpha$-amide. Moreover, two distinct Pen-3a isoforms were also obtained either with an unprocessed glutamine residue or with the natural pyroglutamate at position 1 . Finally, about $50 \%$ of the recombinant molecules were shown to be O-substituted by a dimannosyl moiety on Thr- 6 and -8 (predicted by Netoglyc 2.0 software as potential

\section{$\underline{\text { A }}$ Mature penaeidins}

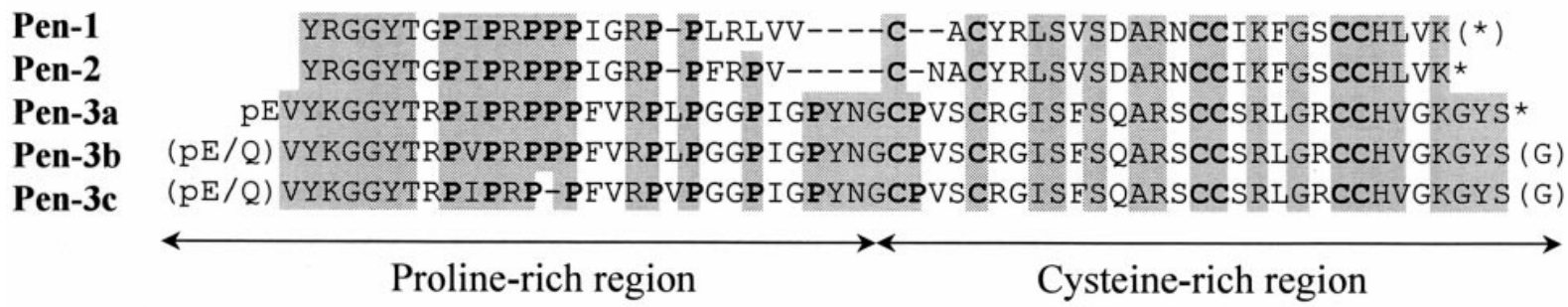

\section{B Penaeidin precursors}

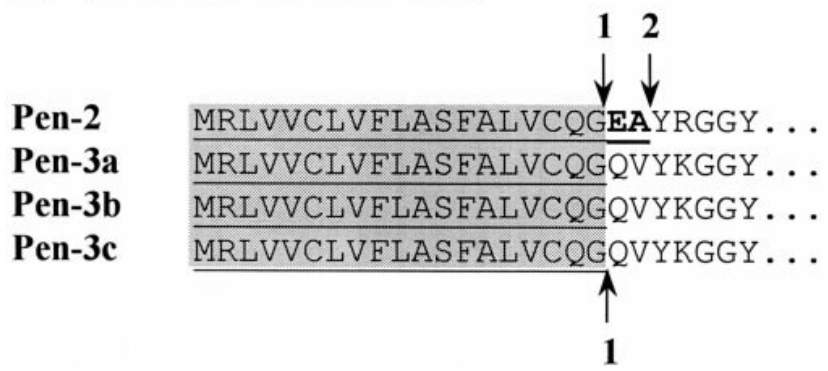

Figure 1. Sequence comparison of penaeidins. $(A)$ Mature penaeidin primary sequences obtained by biochemical characterization (Pen-1, Pen-2, Pen-3a) and/or cDNA cloning data (pen-2, pen-3a, pen-3b, pen-3c) were aligned. Gaps were introduced to optimize the alignment. Cysteine and proline residues are in boldface, and identical amino acids are in boxes. Stars indicate COOH-terminal amidation, and $\mathrm{pE}$ stands for pyroglutamic acid. Assumed posttranslational modifications are in brackets. $(B) \mathrm{The} \mathrm{NH}_{2}$-terminal sequences of penaeidin precursors obtained by amino acid deduction from cDNA sequences were compared. Underlined amino acids show sequences absent in the mature molecules. Arrows indicate the position of the predicted cleavage sites by signal peptidase. 1 and 2 stand respectively for preferential and secondary cleavage sites according to SignalP IV.1 software. Identical residues are in boxes, and the dipeptide Glu-Ala (EA) found in Pen-2 precursor sequence exclusively is in boldface. 
mRNA sequences

Penaeidin-2

Penaeidin-3a

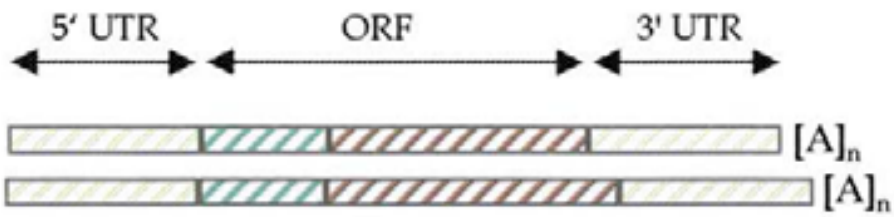

TRANSLATION

\section{Peptide precursors}

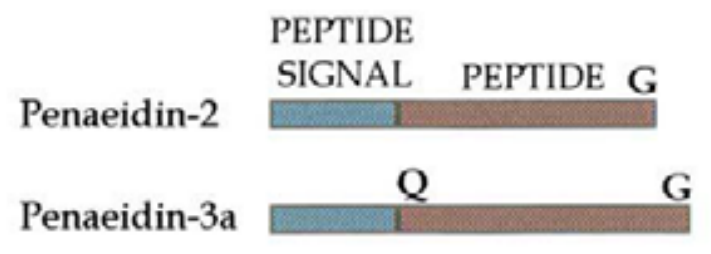

PROCESSING

\section{$\underline{\text { Mature peptides }}$}

Penaeidin-2

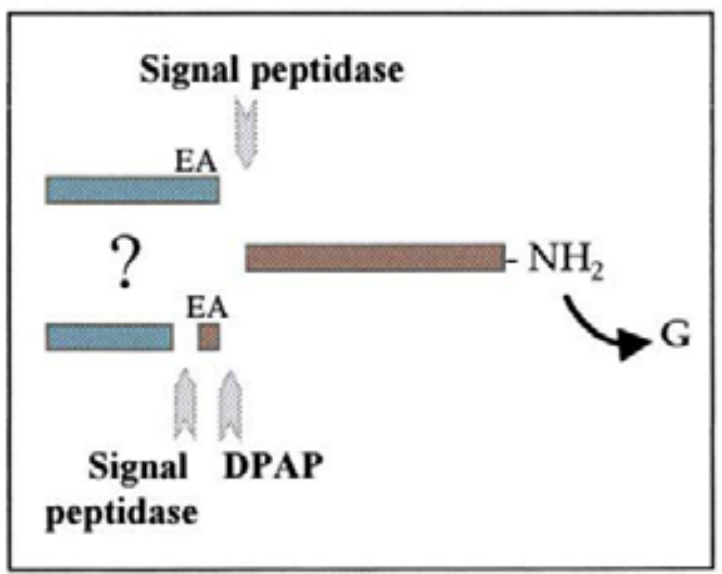

Penaeidin-3a

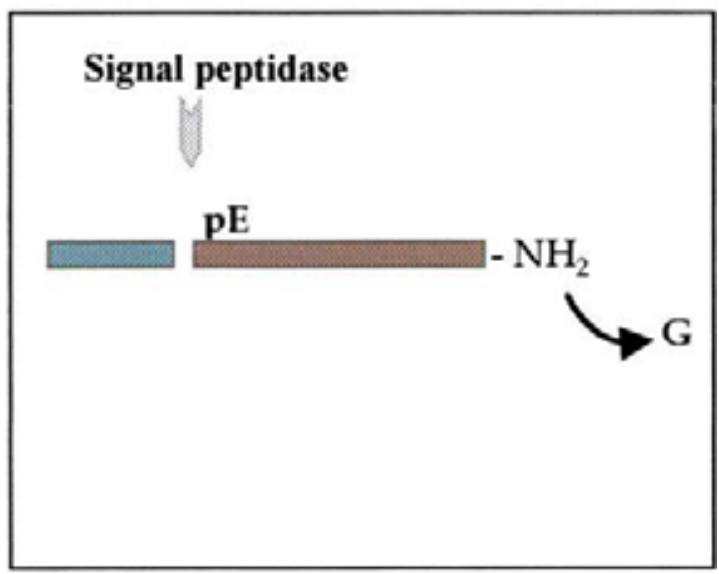

Figure 2. Penaeidin processing in Penaeus vannamei hemocytes. Pen-2 and -3a synthesis from mRNA to mature peptides is represented. After transcription and translation, the two molecules undergo posttranslational modifications including elimination of signal peptide, and $\mathrm{COOH}$-terminal amidation by elimination of a glycine residue. In Pen-3a, an additional step involving formation of a pyroglutamic acid by cyclization of a glutamine residue occurs. Two different processing pathways leading to the removal of the 21 first amino acids of Pen-2 precursor are represented with (i) elimination of a 21-residue signal peptide, or (ii) a two-step process including elimination of a 19-residue signal peptide and subsequent removal of a Glu-Ala dipeptide by a dipeptidyl aminopeptidase (DPAP).

glycosylation sites) for Pen-2 and -3 a, respectively. The availability of the different recombinant molecules allowed investigation of the role of posttranslational modifications in antimicrobial peptide ac- tivity (see below). Finally, because the nonsubstituted recombinant molecules displayed activity similar to native ones, they were further used to enlarge activity spectrum of penaeidin. 


\section{Antimicrobial properties of penaeidins}

Preliminary antimicrobial assays performed with native penaeidins purified to homogeneity showed that penaeidins had both antibacterial and antifungal activities [21]. To determine the activity spectrum in more detail, large quantities of peptides were required and, hence, recombinant penaeidins were employed.

\section{Antifungal activity}

Pen-2 and -3a have broad-spectrum fungicidal activity against filamentous fungi with minimum inhibitory concentrations (MIC) below $10 \mu \mathrm{M}$, but are inactive against yeast such as $S$. cerevisiae or Candida albicans [34]. Interestingly, penaeidins are active against the shrimp pathogen Fusarium oxysporum, which is responsible for infections in different Penaeus species [35, 36], whereas such infections remain unreported in $P$. vannamei. Many phytopathogenic fungal strains such as Nectria haematococca, Alternaria brassicola, Neurospora crassa and Botritys cinerea were shown to be sensitive to the peptides, indicating that penaeidins could have applications in agronomy as therapeutic agents. Penaeidin fungicidal activity against the different strains was shown to be associated with inhibition of spore germination. At lower concentrations, the peptides cause growth inhibition of fungal hyphae, resulting in morphological abnormalities.

\section{Antibacterial activity}

Penaeidin antibacterial activity was found to be rather specific and is essentially directed against Gram-positive bacteria via a strain-specific inhibition mechanism. We observed that depending on the strains penaeidins displayed a rapid killing activity or bacteriostatic properties [34]. This suggests that penaeidins could act through multiple modes of actions. Incubation of Bacillus megaterium strain with Pen-2 or Pen-3 at concentrations 10-fold higher than their MIC values ( $\mathrm{MIC}=2.5-5 \mu \mathrm{M})$ resulted in their immediate killing. On the other hand, a noticeable potent growth inhibition $(\mathrm{MIC}=0.3-0.6 \mu \mathrm{M}$ for Pen-3a) associated with slow killing of the target bacteria $(10 \%$ of bacteria are still alive after a 24-h incubation) was observed on Aerococcus viridans, which is reported to be highly pathogenic in crabs and lobsters, in which it induces rapid septicemia and subsequent death [37]. Finally, penaeidins were found to display little activity against Gram-negative strains, including the Vibrio species commonly responsible for many crustacean bacterial infections [38, 39].

Despite better efficiency of Pen-3a on most of the strains tested, similar activity spectra and inhibition mechanisms are observed for both Pen-2 and -3a (at concentrations above the MIC, sensitive strains are killed or inhibited with identical time courses). No synergistic effect was observed between the two molecules against sensitive microorganisms. Nevertheless, because other antimicrobial peptides are likely to be present in shrimp, as evidenced by reversed-phase high-performance liquid chromatography (HPLC) of hemolymph acid extracts and subsequent antimicrobial assays [21], such a synergism could exist between penaeidins and other putative antimicrobial peptides present in shrimp hemolymph, in a manner similar to that proposed in other organisms [13, 24, 40, 41].

\section{Effect of posttranslational modifications on bioactivity of penaeidins}

The role of posttranslational modifications observed in native penaeidins (COOH-terminal amidation and $\mathrm{NH}_{2}$-terminal pyroglutamic acid in Pen-3a) was studied with the set of penaeidin variants obtained by recombinant expression (see above). These modifications were found to have little effect on penaeidin antimicrobial properties [34], but possibly they increase the stability of penaeidins, which are highly resistant to proteolysis [21].

$\mathrm{NH}_{2}$-terminal blocking has no effect on penaeidin antimicrobial properties, as reported for the plant antifungal peptide Rs-AFP2 isolated from Raphanus sativus [33]. Moreover, when COOH-terminal amidation is replaced by an extra glycine residue, penaeidin antifungal properties are unaltered, and antibacterial activity is decreased only twofold. Similar to reports on insect cecropins [42, 43], the partial loss of antibacterial activity that occurs in nonamidated penaeidins may be due to the loss of a positive charge at the $\mathrm{COOH}$-terminus, and consequently to less efficient interaction of the peptide with bacterial membranes.

Several antimicrobial peptides, such as the proline-rich Drosophila melanogaster drosocin [44] or the glycinerich Phormia terranovae diptericin [45], occur naturally as $\mathrm{O}$-glycosylated molecules, and substitution is necessary for full biological activity of these peptides [1]. In contrast, no member of the penaeidin family has been extracted from shrimp in a substituted form, and O-glycosylation of recombinant penaeidins induced a two to fourfold decrease in penaeidin activity against half of the microorganisms tested [34]. In drosocin, it was proposed that the carbohydrate moiety might be involved in conformational changes beneficial to the stereospecific recognition of a target molecule on bacterial membranes [44]. However, a recent report by McManus et al. on the three-dimensional (3D) structure of drosocin revealed no substantial difference in the structure of drosocin with or without the sugar part [46]. The eluci- 
dation of the 3D structure of penaeidin would clarify the possible implication of the glycan part in destabilizing the secondary structure of the recombinant O-glycosylated molecule.

\section{Role of penaeidin $\mathrm{NH}_{2}$ - and $\mathrm{COOH}-t e r m i n a l$ regions}

Penaeidins combine two domains in their overall structure, one proline-rich and the other cysteine-rich, usually observed in distinct groups of antimicrobial peptides. Similarly, the big horseshoe crab defensin [47] is composed of (i) a $\mathrm{NH}_{2}$-terminal domain without evident homologies with other antimicrobial peptides and displaying anti-Gram-positive activity, and (ii) a $\mathrm{COOH}$-terminal domain with three disulfide bonds displaying anti-Gram-negative properties. Thus, the overall biological activity of penaeidin may be associated with distinct properties of their two characteristic regions.

\section{$\mathrm{NH}_{2}$-terminal region}

The penaeidin $\mathrm{NH}_{2}$-terminal proline-rich region shares sequence similarities with other proline-rich antimicrobial peptides. In particular, penaeidins display $70-76 \%$ identity over 17 residues, with the $6.5-\mathrm{kDa}$ peptide isolated from the crab C. maenas [19]. This region also shares $60 \%$ identity over 10 residues with the bovine bactenecin-7 (Bac7), and contains a Pro-Ile-Pro-ArgPro motif, repeated three times in the Bac7 sequence [48] (fig. 3a).

A synthetic peptide corresponding to the $\mathrm{NH}_{2}$-terminal proline-rich domain of penaeidin (residues 1-20) was produced and found to be inactive against both bacterial and fungal strains [unpublished data]. Similar observations were also made with the Bac7 repeated peptide homologous to the penaeidin $\mathrm{NH}_{2}$-terminal region [49]. Since it was demonstrated that Bac7 is able to tightly bind artificial membranes through electrostatic interaction, we can speculate that the penaeidin prolinerich region is similarly involved more in target cell interaction than in direct antimicrobial activity. This is also consistent with the spectrum of penaeidin antimicrobial activity, which is directed mainly against fungi and Gram-positive bacteria [34], in contrast to most of the known proline-rich peptides, which have essentially anti-Gram-negative properties [1].

\section{COOH-terminal region}

Since the penaeidin $\mathrm{NH}_{2}$-terminal region failed to exhibit antimicrobial activity, it is tempting to speculate that the $\mathrm{COOH}$-terminal region mediates penaeidin antimicrobial properties. In addition, most of the antimi- crobial peptides with intramolecular disulfide bridges, such as defensins, have antimicrobial properties similar to those observed for penaeidins. Defensins are found in a large range of animal species, both in vertebrates and invertebrates [50], and show a highly conserved cysteine motif. On the basis of the position and bonding of the six conserved cysteine residues, defensins from vertebrates are divided into two categories, $\alpha$ - and $\beta$-defensins [51], whereas insect defensins fit into a distinct group [52]. The penaeidin cysteine-spacing - which consists of two cysteine doublets conserved between the different molecules, and two additional cysteine residues separated by one, two and three amino acids in Pen-1, -2 and -3 , respectively (fig. 1a) - does not correspond to any of the motifs hitherto described, and the cysteine connectivity remains to be determined by 3D-structure analysis.

The penaeidin $\mathrm{COOH}$-terminal region was found to display partial conservation of a repeated motif common to several chitin-binding proteins of the hevein family, such as wheat germ agglutinin [53] (fig. 3b). Interestingly, this feature is also found in two other antimicrobial peptides: (i) the plant antimicrobial protein-2 (Ac-AMP2) isolated from Amaranthus caudatus $[54,55]$, which also contains six cysteine residues and has significant sequence similarities with penaeidins (fig. 3b); and (ii) tachycitin (10 cysteines), a peptide isolated from horseshoe crab hemocytes [41]. Their partial conservation of a chitin-binding motif is associated with an ability to attach chitin (a component of the fungal cell wall) tightly, and as penaeidins, these peptides display both antibacterial and antifungal activities. Chitin binding has also been demonstrated for intact penaeidins but was absent for their synthetic $\mathrm{NH}_{2}$-terminal domain (20 amino acids) [56]. Thus we believe that binding of penaeidins to chitin, and consequently their antifungal activity, is mediated by their $\mathrm{COOH}-$ terminal domain.

\section{Penaeidin chimeric-like structure}

Penaeidins consist of two structurally distinct regions whose functions remain unstudied. Two families of plant proteins were shown to have a similar chimericlike structure: (i) the tobacco extracellular matrix proteins CELPs - for cysteine-rich extensin-like protein [57] - that have a proline-rich extensin-like domain and a cysteine-rich domain with a highly charged $\mathrm{COOH}-$ terminus; and (ii) the potato lectins, which have an extensin domain rich in glycosylated hydroxyproline residues, fused with a lectin domain containing partial conservation of a repeated motif common to the chitinbinding proteins of the hevein family [53]. These chimeric proteins might be multifunctional [57] with roles in plant growth, development and defense. Inter- 
estingly, penaeidins display homologies with CELPs (fig. 4) and thus, as proposed for these molecules, penaeidin overall structure could also be associated with several functions. In particular, as crustaceans also contain chitin as a major cuticle component, penaeidin chitin-binding ability could also be involved in wound healing or chitin assembly during the moulting process. Experimental comparison of the two characteristic penaeidin regions in terms of activity should help to clarify their involvement in the properties of the entire molecule but also to aid in understanding the role of penaeidins in the shrimp defense system.

\section{A Penaeidin $\mathrm{NH}_{2}$-terminal region}

\section{C. maenas 6,5 kDa peptide}

Penaeidin-1

Penaeidin-2

Penaeidin-3a

Penaeidin-3b

Penaeidin-3c

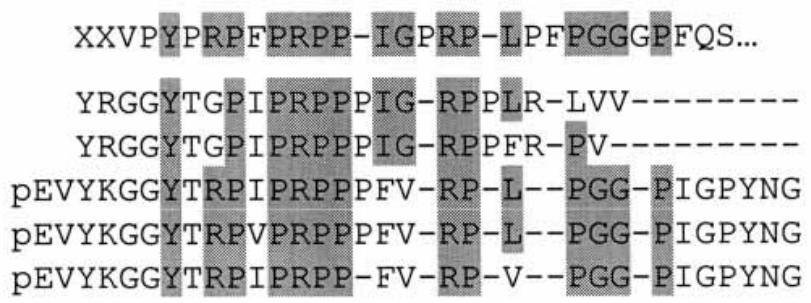

Bactenecin-7

RRIRPRPPRLPRPRPRPLPFPRPGPRPIPRPLPFPRPGPR-PIPRPLPFPRPGPRPIPRP

Penaeidin-1

Penaeidin-2

Penaeidin-3a

Penaeidin-3b

Penaeidin-3c

\section{B Penaeidin $\mathrm{COOH}$-terminal region}

YRGGYTGPIPRPPPIGRPPLR-LVV----

YRGGYTGPIPRPPPIGRPPER-PV-----

PEVYKGGYTRPIPRPPPEVRPLPGGPIGPYNG

PEVYKGGYTRPVPRPPPFVRPLPGGPIGPYNG

PEVYKGGYTRPIPRP-PFVRPVPGGPIGPYNG

\begin{tabular}{|c|c|}
\hline & MOTIF \\
\hline itin-binding domain & 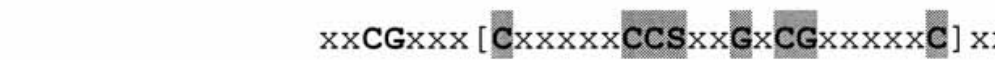 \\
\hline Penaeidin-3a & GGPIGPYNGCPV--SC--RGISFSQARSCCSRLGRCCHVGKGYS* \\
\hline Ac-AMP2 & V-GECV-RGRCPS--GMCCSQFGYC-GKGPKYCG \\
\hline Tachycitin & RCGRYSPCLDDGPNVN-LYSCCS-FYNC-HKCLARLE \\
\hline Hevein & AEQCGR-----QAGGKLCPNNLCCSQWGWC-GSTDEYCS \\
\hline WGA (D3) & GGPCRADIKCG---SQQ--SGGKLCPNNLCCSQWGFC-GLGSEFCG \\
\hline WGA (D4) & KPCG----KDAGGRVCTNNYCCSKWGSC-GIGPGYCG \\
\hline PL-C & -----KEFGGKECHDELCCSQYGWC-GNSDGHC \\
\hline
\end{tabular}

Figure 3. Sequence comparison of penaeidin $\mathrm{NH}_{2}$ - and $\mathrm{COOH}$-terminal regions with other peptides/proteins, $(A)$ The penaeidin $\mathrm{NH}_{2}$-terminal proline-rich region was aligned with the $\mathrm{NH}_{2}$-terminal sequence of the Carcinus maenas 6.5-kDa peptide [19], and with bactenecin-7 from bovine neutrophils [48]. Gaps were introduced to optimize the alignment. Identical amino acids and conservative replacements are shaded, and the PIPRP motif, repeated three times in bactenecin-7, is in boldface. Nonidentified amino acids are represented by $\mathrm{X} .(B)$ The penaeidin $\mathrm{COOH}$-terminal sequence was compared with the chitin binding domain (boldface, amino acids) of lectins and antifungal peptides isolated from plants. The amino acids belonging to the consensus pattern are shaded as well as amino acid conservative replacements. Stars indicate a carboxy-terminal amidation. The two antimicrobial proteins Ac-AMP2 and tachycitin, which also display partial conservation of the chitin binding motif, are included in the alignment. The following sequences are aligned: Ac-AMP2, Amaranthus caudatus, antimicrobial peptide [54]; tachycitin, antimicrobial peptide from Tachypleus tridentatus [41]; hevein, antifungal peptide from Hevea brasiliensis and WGA, wheat germ agglutinin [73]; PL-C, Phytolacca americana lectin C [74]. 


$\begin{array}{lr}\text { Penaeidin-3 } & \text { PEVYKGGYTRPIPRPPPEV--RPLPGGPIGPYNGCP } \\ \text { CELP } & \text { RPYPCPPPRP--RPCPPPPPPP---CP } \\ \text { PTL1 } & \text { RPMPKPPSTIPKRPPPKGPLKPP } \\ \text { EXTN_SORVU } & (1) \\ \text { EXTN_SORVU } & \text { YGGGYPTPTPKPPAKGP-KP } \\ \text { PR33_DAUCA } & \text { YTPPVSHTPSSPP--PP } \\ \text { YTPPVHKPPSEY--KPP }\end{array}$

Figure 4. Comparison of penaeidin $\mathrm{NH}_{2}$-terminal domain with plant extensins. CELP, cysteine-rich extensin-like protein from Nicotiana tabacum [57]; PTL1, extensin-like protein from Antirrhinum majus [75]; EXTN SORVU ${ }^{(1)}$ residues 30-48 and EXTN_SORVU ${ }^{(2)}$ residues 263-279, extensin from Sorghum vulgare [76]; PR33_DAUCA, extensin from Daucus carota [77]. Conservative replacements of identical amino acids are shaded. A VYK motif involved in intermolecular cross-links in extensins [78] is boxed.

\section{Penaeidin expression and immune function}

In arthropods, antimicrobial peptide synthesis and tissue localization were studied in horseshoe crabs, and regulation of their gene expression in response to injuries was studied in insects. Our recent studies devoted to penaeidins provide information about the immune reaction mediated by antimicrobial peptides in crustaceans.

\section{Expression in unchallenged animals}

Comparison of penaeidin expression levels in various cells and tissues has demonstrated that hemocytes are the main site for penaeidin synthesis. The intense hybridization signals observed by Northern blot experi- ments [56] were consistent with the high percentage of positive clones previously obtained during screening of the shrimp cDNA library (168 positive clones over $500,000 \mathrm{pfu}$ ) [21]. Besides their active transcription of penaeidin genes, hemocytes were also shown to store peptides. In fact, penaeidins represent the bulk of proteinaceous material observed by reversed-phase HPLC [21] in acid extracts from organelle-rich hemocyte fractions, and some hemocyte populations are highly reactive to penaeidin immunostaining [56].

On the basis of morphological observations, shrimp hemocytes are classified into two distinct groups, (i) the hyaline cells (devoid of cytoplasmic electron-dense granules), and (ii) the hemocytes, with large granules (LGH) or small granules (SGH) [58]. Immunogold experiments clearly demonstrated that penaeidins are contained in the cytoplasmic granules of the LGH (fig. 5) and to a lesser extent in SGH [56], but that hyaline cells are devoid of stored penaeidins. Thus it is believed that after synthesis penaeidins undergo different posttranslational modifications in the endoplasmic reticulum (elimination of the signal peptide) and in the Golgi (COOH-terminal amidation and $\mathrm{NH}_{2}$-terminal cyclization of a glutamine residue in Pen-3a), before being addressed and stored in hemocyte cytoplasmic granules. This scheme is similar to that proposed in horseshoe crab hemocytes [5] or mammalian neutrophils [59, 60]. In other respects, the absence of penaeidin immunoreactivity in hyalinocytes does not indicate that they do not express the peptides. This cell type could directly secrete the newly synthesized penaeidins into the plasma as do antimicrobial peptides in the insect fat body cells [61].
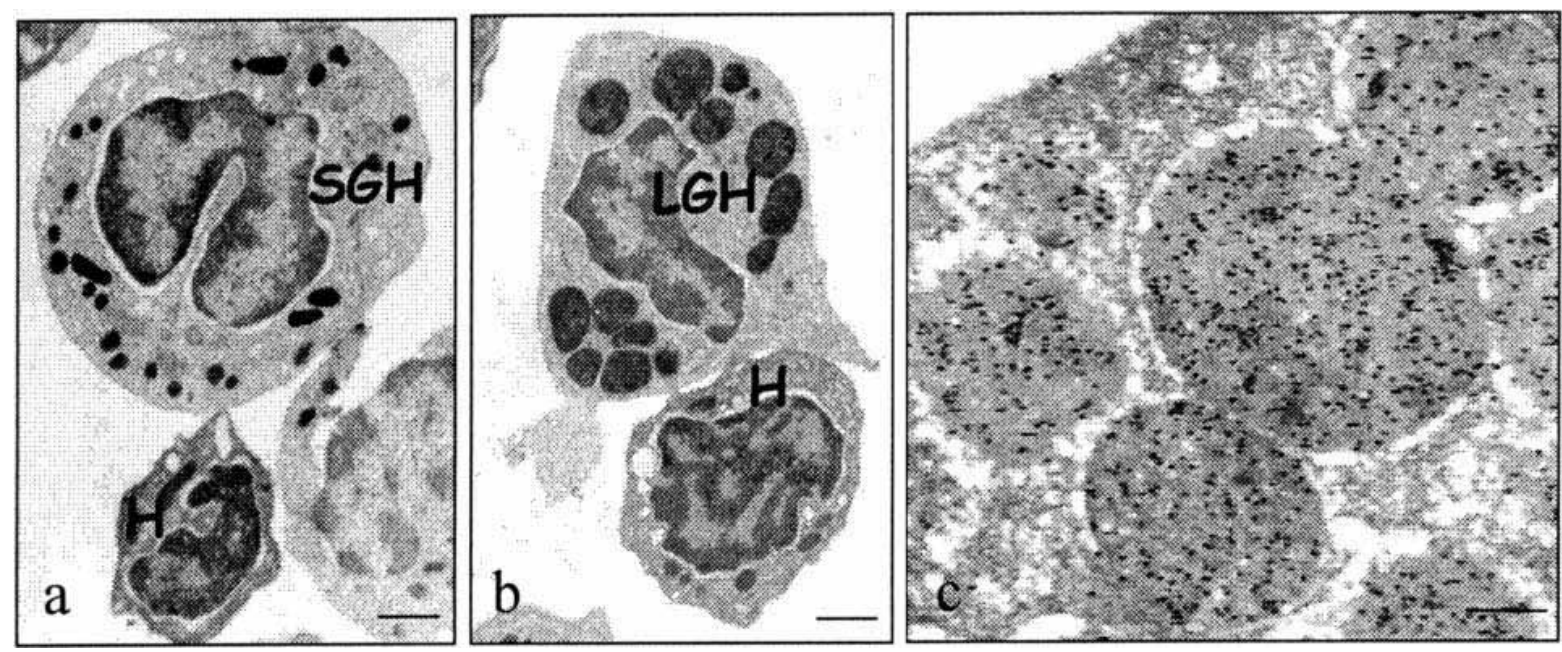

Figure 5. Ultrastructural localization of penaeidins in Penaeus vannamei hemocytes. Transmission electron micrographs of (a) granular hemocyte with large granules $(\mathrm{LGH})$ and hyaline hemocyte $(\mathrm{H})$ and $(b)$ granular hemocyte with small granules $(\mathrm{SGH})(\mathrm{bar}, 1 \mu \mathrm{m})$. $(c)$ Immunolocalization of penaeidins by immunogold labelling in cytoplasmic granules of haemocyte with large granules (bar, $500 \mathrm{~nm}$ ). 

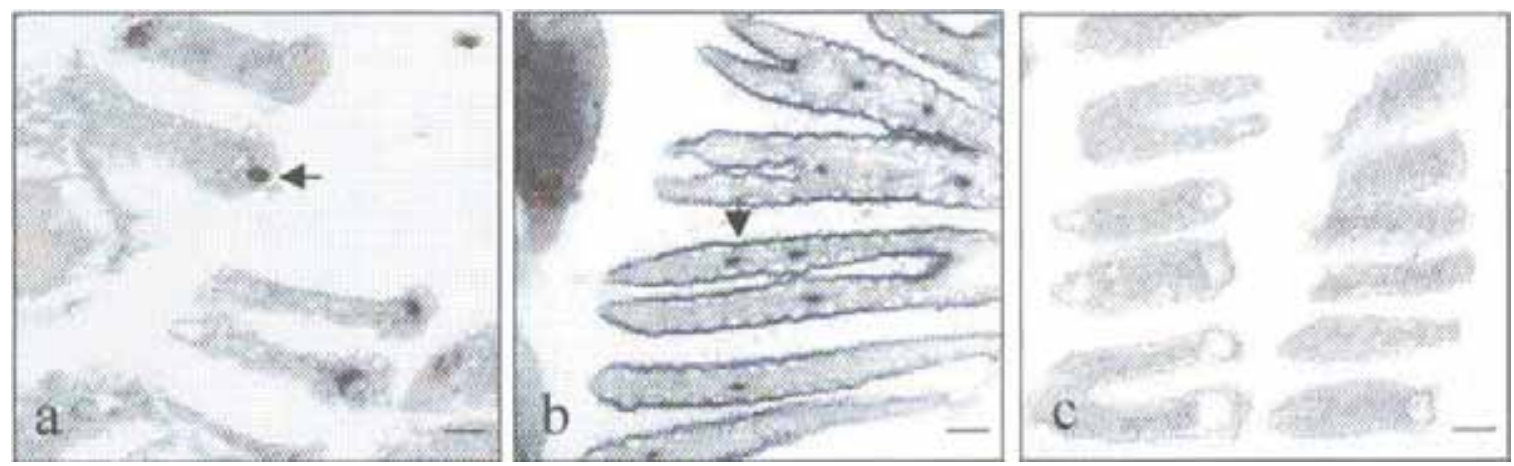

Figure 6. Penaeidin immunoreactivity in gill filaments. (a) Immunoreactivity is observed in hemocytes located in gill efferent blood vessels of unchallenged shrimp. (b) Penaeidin immunoreactivity, as observed after microbial challenge (24 h), is found at the level of gill cuticle when $(c)$ no labelling is noticed in control (nonimmune serum) (bar, $10 \mu \mathrm{m}$ ).

In situ hybridization experiments on hemocyte populations should help to resolve this issue.

\section{Expression following microbial challenge}

In vivo microbial challenge experiments (injection of heat-killed bacteria and fungi in shrimp) demonstrated that penaeidin transcription is not upregulated in shrimp hemocytes [56] as observed for antimicrobial peptides in the insect fat body [12], but induces an increase in the penaeidin concentration in plasma. Thus, although other tissues could contribute to penaeidin release into the bloodstream, we can speculate that along with other components, penaeidins are released from hemocyte cytoplasmic granules in response to microbial stimulation. Similar release of antimicrobial substances has previously been shown by in vitro stimulation of horseshoe crab hemocytes (in contact with bacterial endotoxins) [62]. Thus, as proposed for Limulus, regulated exocytosis of antimicrobial peptides from shrimp granular hemocytes could help to circumvent microbial infections by increasing plasma antimicrobial activity in the contact areas between microbes and hemocytes.

Secretion of antimicrobial peptides was also described for human neutrophils that release granule-stored cathelicidin hCAP-18 into the bloodstream upon challenge [63]. Within the same cells, the $\alpha$-defensins HNP1 -4 are stored in a nonsecretory granule type, and act intracellularly after fusion of the granules to the microbe-containing phagosome [64]; penaeidins could act in a similar manner during phagocytic and postphagocytic events. Granule-containing hemocytes are phagocytic cells [65], and they are also involved in nodule formation, where small-granule hemocytes (found in the most central part of the nodule) undergo phagocytosis and lose their cytoplasmic granules [66] Thus penaeidin release into the bloodstream could result from localized degranulation events within multicellular structures where high penaeidin concentrations could be reached in contact with the microbes.

Besides the high transcription levels observed in hemocytes, significant concentrations in penaeidin messenger RNA (mRNA) were observed by Northern blot analysis in tissues rich in hemocytes such as the heart or gills. It was consequently supposed that in these tissues the observed signals were due to the hemocyte ability to infiltrate tissues. Penaeidin immunocytochemical patterns observed in response to microbial challenge are consistent with this hypothesis. In gills, in particular, whereas penaeidins are immunolocalized in granulocytes observed in the filament blood vessels of unchallenged shrimp, a noteworthy immunoreactivity appears on the gill cuticle surfaces following a microbial stimulation (fig. 6), and no more penaeidin-reactive hemocytes are observed in the efferent blood vessels [56]. Hence, after release into the plasma, penaeidins could attach to the shrimp cuticle through their chitin-binding properties and ensure optimal protection of the whole animal.

Recently it has been demonstrated in insects that the surface epithelia of gut tissue [67], trachea [68] or reproductive tract [69] synthesize antimicrobial peptides. In addition, the mammalian intestine was also found to produce defensins $[70,71]$. It now appears worthwhile to investigate the expression of penaeidins in epithelial barriers where they could prevent infections from exterior microorganisms. Such a study will be of special importance for a better understanding of crustacean immune defense reactions in conjunction with their aquatic environment, which is rich in potentially pathogenic microorganisms. 


\section{Conclusion}

The recent discovery of antimicrobial peptides in crustaceans provides new clues for fundamental understanding of crustacean immunity. The studies on the shrimp penaeidins have largely contributed to this knowledge as they are the first antimicrobial peptides fully characterized in a crustacean and for which expression studies were performed.

The well-established involvement of hemocytes in shrimp defense reinforces the hypothesis of a major role of penaeidins as effectors of shrimp immune defense. On the one hand, by their presence and their ability to migrate to tissues, hemocytes are likely to insure penaeidin transport to sites exposed to environmental pathogenic microorganisms. However, even though we still do not have evidence of such a phenomenon, penaeidin expression in physiological barriers such as epithelia will be an important question to address. These two defense reactions, which would mediate local protection to limit invasion by foreign microbes, are most likely relayed by systemic protection as suggested by penaeidin release into the plasma, thus providing a second line of defense to circumvent microbial infection.

Besides their antimicrobial properties, penaeidins may be involved in additional functions as suggested by their chimeric-like structure. In particular, penaeidin chitinbinding ability may constitute one of the most interesting aspects of these peptides. Not only is this property likely to be essential for penaeidin antifungal activity, but penaeidins could also be involved in chitin assembly or wound healing and as such be essential in shrimp protection during the moulting cycle.

Knowledge of penaeidin functions will be particularly important for further establishment of disease control in penaeid shrimp production [72]. Moreover, from their unusual features both at the structural level and in terms of biological activity, penaeidins could represent, along with other antimicrobial peptides, a new generation of therapeutic agents and find potential applications in aquaculture or agronomy.

Acknowledgements. This work is supported by IFREMER (Institut Français de Recherche et d'Exploitation de la Mer), the CNRS (Centre National de la Recherche Scientifique) and the University of Montpellier 2. It is also part of a collaborative Concerted Action project (IC18CT970209) supported by the European Commission, DG XII, in the program 'International Cooperation with Developing Countries, INCO-DC' (Shrimp Immunity and Disease Control). The authors are grateful to Dr Edith Porter for improving the English of the manuscript.

1 Bulet P., Hetru C., Dimarcq J.-L. and Hoffmann D. (1999) Antimicrobial peptides in insects; structure and function. Dev. Comp. Immunol. 23: 329-344
2 Shai Y. (1998) Mode of action of antibacterial peptides. In: Molecular Mechanisms of Immune Responses in Insects, pp. 111-134, Brey P. T. and Hultmark D. (eds), Chapman and Hall, London

3 Carlsson A., Engstrom P., Palva E. T. and Bennich H. (1991) Attacin, an antibacterial protein from Hyalophora cecropia, inhibits synthesis of outer membrane proteins in Escherichia coli by interfering with omp gene transcription. Infect. Immun. 59: 3040-3045

4 Ganz T. and Lehrer R. I. (1997) Antimicrobial peptides of leukocytes. Curr. Opin. Hematol. 4: 53-58

5 Iwanaga S., Kawabata S.-I. and Muta T. (1998) New types of clotting factors and defense molecules found in horseshoe crab hemolymph: their structures and functions. J. Biochem. 123: $1-15$

6 Dimarcq J. L., Keppi E., Dunbar B., Lambert J., Reichhart J. M., Hoffmann D. et al. (1988) Insect immunity. Purification and characterization of a family of novel inducible antibacterial proteins from immunized larvae of the dipteran Phormia terranovae and complete amino-acid sequence. Eur. J. Biochem. 171: 17-22

7 Mitta G., Hubert F., Noël T. and Roch P. (1999) Myticin, a novel cysteine-rich antimicrobial peptide isolated from hemocytes and plasma of the mussel Mytilus galloprovincialis. Eur. J. Biochem. 264: 1-9

8 Lee I. H., Zhao C., Cho Y., Harwig S. S., Cooper E. L. and Lehrer R. I. (1997) Clavanins, alpha-helical antimicrobial peptides from tunicate hemocytes. FEBS Lett. 400: 158-162

9 Zhao C., Liaw L., Lee I. H. and Lehrer R. I. (1997) cDNA cloning of three cecropin-like antimicrobial peptides (styelins) from the tunicate, Styela clava. FEBS Lett. 412: 144-148

10 Ouellette A. J. and Selsted M. E. (1996) Paneth cell defensins: endogeneous peptide components of intestinal host defense. FASEB J. 10: 1280-1289

11 Engström Y. (1998) Insect immune gene regulation. In Molecular Mechanisms of Immune Responses in Insects, pp. 211-244, Brey P. T. and Hultmark D. (eds), Chapman and Hall, London

12 Hoffmann J. A. and Reichhart J.-M. (1997) Drosophila immunity. Trends Cell Biol. 7: 309-316

13 Iwanaga S. and Kawabata S.-I. (1998) Evolution and phylogeny of defense molecules associated with innate immunity in horseshoe crab. Front. Biosci. 3: D973-D984

14 Stewart J. E. and Zwicker B. M. (1972) Natural and induced bactericidal activities in the hemolymph of the lobster, Homarus americanus: products of hemocyte-plasma interaction. Can. J. Microbiol. 18: 1499-1509

15 Mori K. and Stewart J. E. (1978) The hemolymph bactericidin of the american lobster (Homarus americanus) adsorption and activation. J. Fish. Res. Board Canada 5: 1504-1507

16 White K. N., Ratcliffe N. A. and Rossa M. (1985) The antibacterial activity of haemocyte clumps in the gills of the shore crab, Carcinus maenas. J. Mar. Biol. 65: 857-870

17 Chisholm J. R. S. and Smith V. J. (1992) Antibacterial activity in the haemocytes of the shore crab, Carcinus maenas. J. Mar. Biol. Assoc. U.K. 72: 529-542

18 Chisholm J. R. S. and Smith V. J. (1995) Comparison of antibacterial activity in the hemocytes of different crustacean species. Comp. Biochem. Physiol. 110A: 39-45

19 Schnapp D., Kemp G. D. and Smith V. J. (1996) Purification and characterization of a proline-rich antibacterial peptide, with sequence similarity to bactenecin-7, from the haemocytes of the shore crab, Carcinus maenas. Eur. J. Biochem. 240: $532-539$

20 Khoo L., Robinette D. W. and Noga E. J. (1999) Callinectin, an antibacterial peptide from blue crab, Callinectes sapidus, hemocytes. Marine Biotechnol. 1: 44-51

21 Destoumieux D., Bulet P., Loew D., Van Dorsselaer A., Rodriguez J. and Bachère E. (1997) Penaeidins: a new family of antimicrobial peptides in the shrimp Penaeus vannamei (Decapoda). J. Biol. Chem. 272: 28398-28406

22 Nakamura T., Furunaka H. and Miyata T. (1988) Tachyplesin, a class of antimicrobial peptide from the hemocytes of 
the horseshoe crab (Tachypleus tridentatus). Isolation and chemical structure. J. Biol. Chem. 263: 16709-16713

23 Miyata T., Tokunaga F., Yoneya T., Yoshikawa K., Iwanaga S., Niwa M. et al. (1989) Antimicrobial peptides, isolated from horseshoe crab hemocytes, tachyplesin II, and polyphemusins I and II: chemical structures and biological activity. J. Biochem. (Tokyo) 106: 663-668

24 Casteels P., Ampe C., Jacobs F. and Tempst P. (1993) Functional and chemical characterization of Hymenoptaecin, an antibacterial polypeptide that is infection-inducible in the honeybee (Apis mellifera). J. Biol. Chem. 268: 7044-7054

25 Selsted M. E., Tang Y. Q., Morris W. L., McGuire P. A., Novotny M. J., Smith W. et al. (1993) Purification, primary structures and antibacterial activities of beta-defensins, a new family of antimicrobial peptides from bovine neutrophils. J. Biol. Chem. 268: 6641-6648

26 Nielsen H., Engelbrecht J., Brunak S. and von Heijne G. (1997) Identification of prokaryotic and eukaryotic signal peptides and prediction of their cleavage sites. Protein Eng. 10: $1-6$

27 Kreil G. (1990) Processing of precursors by dipeptidylaminopeptidases: a case of molecular ticketing. Trends Biochem. Sci. 15: 23-26

28 Boman H. G., Faye I., Gudmunssson G. H., Lee J. Y. and Lidholm D. A. (1991) Cell-free immunity in Cecropia. A model system for antibacterial proteins. Eur. J. Biochem. 201: 23-31

29 Shigenaga T., Muta T., Toh Y., Tokunaga F. and Iwanaga S. (1990) Antimicrobial tachyplesin peptide precursor cDNA cloning and cellular localization in the horseshoe crab (Tachypleus tridentatus). J. Biol. Chem. 265: 21350-21354

30 Valore E. V., Martin E., Harwig S. S. and Ganz T. (1996) Intramolecular inhibition of human defensin HNP-1 by its propiece. J. Clin. Invest. 97: 1624-1629

31 Reichhart J.-M., Petit I., Legrain M., Dimarcq J.-L., Keppi E., Lecocq J.-P. et al. (1992) Expression and secretion in yeast of active insect defensin, an inducible antibacterial peptide from the flesh fly Phormia terranovae. Invert. Reprod. Dev. 21: $15-24$

32 Michaud L., Felhbaum P., Moniatte M., Van Dorsselaer A., Reichhart M. and Bulet P. (1996) Determination of the disulfide array of the first inducible antifungal peptide from insects: drosomycin from Drosophyla melanogaster. FEBS Lett. 395: $6-10$

33 Vilas Alves A. L., de Samblanx G. W., Terras F. R. G., Cammue B. P. A. and Broekaert W. F. (1994) Expression of functional Raphanus sativus antifungal protein in yeast. FEBS Lett. 348: 228-232

34 Destoumieux D., Bulet P., Strub J.-M. and Bachère E. (1999) Recombinant expression and range of activity of penaeidins, antimicrobial peptides from penaeid shrimp. Eur. J. Biochem. 266: $335-346$

35 Rhoobunjongde W., Hatai K., Wada S. and Kubota S. S. (1991) Fusarium moniliforme (Sheldon) isolated from gills of Kuruma prawn Penaeus japonicus (Bate) with black gill disease. Nippon Suisan Gakkaishi 57: 629-635

36 Momoyama K. (1987) Distributions of the hyphae in Kuruma shrimp, Penaeus japonicus, infected with Fusarium solani. Fish Pathol. 22: $15-23$

37 Cornick J. W. and Stewart J. E. (1968) Interaction of the pathogen Gaffkya homari with natural defense mechanisms of Homarus americanus. J. Fisher. Res. Board Canada 25: 695709

38 Song Y. L. and Lee S. P. (1993) Characterization and ecological implication of luminous Vibrio harveyi isolated from tiger shrimp (Penaeus monodon). Bull. Inst. Zool. 32: 217-220

39 Roberston P. A. W., Calderon J., Carrerra L., Stark J. R., Zherdmant M. and Austin B. (1998) Experimental Vibrio harveyi infections in Penaeus vannamei larvae. Dis. Aquat. Org. 32: $151-155$

40 Cammue B. P., De Bolle M. F., Schoofs H. M., Terras F. R., Thevissen K., Osborn R. W. et al. (1994) Gene-encoded antimicrobial peptides from plants. Ciba Found Symp. 186: $91-106$
41 Kawabata S. I., Nagayama R., Hirata M., Shigenaga T., Agarwala K. L., Saito T. et al. (1996) Tachycitin, a small granular component in horseshoe crab hemocytes, is an antimicrobial protein with chitin-binding activity. J. Biochem. (Tokyo) 120: 1253-1260

42 Li Z. Q., Merrifield R. B., Boman I. A. and Boman H. G. (1988) Effects on electrophoretic mobility and antibacterial spectrum of removal of two residues from synthetic sarcotoxin Ia and addition of the same residues to cecropin B. FEBS Lett. 231: 299-302

43 Hara S., Taniai K., Kato Y. and Yamakawa M. (1994) Isolation and alpha-amidation of the non-amidated form of cecropin D from the larvae of Bombyx mori. Comp. Biochem. Physiol. 108B: 303-308

44 Bulet P., Urge L., Ohresser S., Hetru C. and Otvos L. (1996) Enlarged scale chemicals synthesis and range of activity of drosocin, an O-glycosylated peptide of Drosophila. Eur. J. Biochem. 238: 64-69

45 Bulet P., Hegy G., Lambert J., Van Dorsselaer A., Hoffmann J. A. and Hetru C. (1995) Insect immunity. The inducible antibacterial peptide diptericin carries two O-glycans for biological activity. Biochemistry 34: 7394-7400

46 McManus A. M., Otvos L., Hoffmann R. and Craik D. J. (1999) Conformational studies by NMR of the anti-microbial peptide drosocin and its nonglycosylated derivative: effects of glycosylation on solution conformation. Biochemistry 38: $705-714$

47 Saito T., Kawabata S., Shigenaga T., Takayenoki Y., Cho J., Nakajima H. et al. (1995) A novel big defensin identified in horseshoe crab hemocytes: isolation, amino acid sequence and antibacterial activity. J. Biochem. (Tokyo) 117: 1131-1137

48 Gennaro R., Skerlavaj B. and Romeo D. (1989) Purification, composition and activity of two bactenecins, antibacterial peptides of bovine neutrophils. Infect. Immun. 57: 3142-3146

49 Tani A., Lee S., Oishi O., Aoyagi H. and Ohno M. (1995) Interaction of the fragments characteristic of bactenecin-7 with phospholipid bilayers and their antimicrobial activity. J. Biochem. 117: 560-565

50 Lehrer R. I. and Ganz T. (1999) Antimicrobial peptides in mammalian and insect host defence. Curr. Opin. Immunol. 11: 23-27

51 Ganz T. and Lehrer R. (1998) Antimicrobial peptides of vertebrates. Curr. Opin. Immunol. 10: $41-44$

52 Dimarcq J.-L., Bulet P., Hétru C. and Hoffmann J. A. (1998) Cysteine-rich antimicrobial peptides in invertebrates. Biopolymers 47: 465-477

53 Allen A. K., Bolwell G. P., Brown D. S., Sidebottom C. and Slabas A. R. (1996) Potatoe lectin: a three domain glycoprotein with novel hydroxy-proline-containing sequences and sequence similarity to wheat-germ agglutinin. Int. J. Biochem. Cell Biol. 28: 1285-1291

54 Broekaert W. F., Maerien W., Terras F. R. G., De Bolle M. F. C., Proost P., Van Damme J. et al. (1992) Antimicrobial peptides from Amaranthus caudatus seeds with sequence homology to the cysteine/glycine-rich domain of chitin-binding proteins. Biochemistry 31: 4308-4314

55 De Bolle M. F., David K. M., Rees S. B., Vanderleyden J., Cammue B. P. and Broekaert W. F. (1993) Cloning and characterization of a cDNA encoding an antimicrobial peptide chiting-binding protein, from amaranth, Amaranthus caudatus. Plant Mol. Biol. 22: 1187-1190

56 Destoumieux D., Munoz M., Cosseau C., Rodriguez J., Bulet P., Comps M. et al. (1999) Penaeidins, antimicrobial peptides with chitin-binding activity, are produced and stored in shrimp granuloccytes and released after microbial challenge. J. Cell Sci. 113: $461-469$

57 Wu H.-M., Zou J., May B., Qing G. and Cheung A. Y. (1993) A tobacco gene family for flower cell wall proteins with a proline-rich domain and a cysteine-rich domain. Proc. Natl. Acad. Sci. USA 90: 6829-6833

58 Martin G. G. and Hose J. E. (1992) Vascular elements and blood (hemolymph). In: Microscopic Anatomy of Invertebrates, vol. 10, pp. 117-146, Harrison F. W. and Hurnes A. G. (eds), Wiley, New York 
59 Valore E. V. and Ganz T. (1992) Posttranslational processing of defensins in immature human myeloid cells. Blood 79: $1538-1544$

60 Ganz T., Liu L., Valore E. V. and Oren A. (1993) Posttranslational processing and targeting of transgenic human defensin in murine granulocytes, macrophage, fibroblast and pituitary adenoma cell lines. Blood 82: 641-650

61 Meister M., Lemaitre B. and Hoffmann J. A. (1997) Antimicrobial peptide defense in Drosophila. BioEssays 19: 10191026

62 Toh Y., Mizutani A., Tokunaga F., Muta T. and Iwanaga S. (1991) Morphology of the granular hemocytes of the japanese horseshoe crab Tachypleus tridentatus and immunocytochemical localization of clotting factors and antimicrobial substances. Cell Tissue Res. 266: $137-147$

63 Shi J. and Ganz T. (1998) The role of protegrins and other elastase-activated polypeptides in the bactericidal properties of porcine inflammatory fluids. Inf. Immun. 66: 3611-3617

64 Selsted M. E. and Ouellette A. J. (1995) Defensins in granules of phagocytic and nonphagocytic cells. Trends Cell Biol. 5: $114-119$

65 Hose J. E. and Martin G. G. (1989) Defense functions of granulocytes in the ridgeback prawn Sicyonia ingentis. J. Invert. Pathol. 53: 335-346

66 Martin G. G., Kay J., Poole D. and Poole C. (1998) In vitro nodule formation in the ridgeback prawn, Sycionia ingentis, and the american lobster, Homarus americanus. J. Invert. Pathol. 117: 155-168

67 Lehane M. J., Wu D. and Lehane S. M. (1997) Midgut-specific immune molecules are produced by the blood-sucking insect Stomoxys calcitrans. Proc. Natl. Acad. Sci. USA 94: $11502-11507$

68 Ferrandon D., Jung A. C., Criqui M. C., Lemaitre B., Uttenweiler-Joseph S., Reichhart J.-M. et al. (1997) A drosomycinGFP reporter transgene reveals a local immune response in
Drosophila that is not dependent on the Toll pathway. EMBO J. 17: $1217-1227$

69 Marchini D., Giordano P. C., Amons R., Bernini L. F. and Dallai R. (1993) Purification and primary structure ceratotoxin $\mathrm{A}$ and $\mathrm{B}$, two antibacterial peptides from the female reproductive accessory glands of the medfly Ceratitis capitata (Insecta: Diptera). Insect Biochem. Molec. Biol. 23: 591-598

70 Ouellette A. J., Greco R. M., James M., Frederick D. Naftilan J. and Fallon J. T. (1989) Developmental regulation of cryptdin, a corticostatin/defensin precursor mRNA in mouse small intestinal crypt epithelium. J. Cell Biol. 108: 1687-1695

71 Porter E. M., Liu L., Oren A., Anton P. A. and Ganz T. (1997) Localization of human intestinal defensin 5 in Paneth cell granules. Infect Immun. 65: 2389-2395

72 Bachère E. (1999) Shrimp immunity and disease control. Aquaculture, in press

73 Raikhel N. V., Lee H.-I and Broekaert W. F. (1993) Structure and functions of chitin-binding proteins. Ann. Rev. Plant Physiol. Plant Mol. Biol. 44: 591-615

74 Yamaguchi K., Mori A. and Funatsu G. (1995) The complete amino acid sequence of lectin-C from the roots of pokeweed (Phytolacca americana). Biosci. Biotechnol. Biochem. 59: 1384-1385

75 Baldwin T. C., Coen E. S. and Dickinson H. G. (1992) The ptl1 gene expressed in the transmitting tissue of Antirrhinum encodes an extensin-like protein. Plant J. 2: 733-739

76 Raz R., Cretin C., Puigdomenech P. and Martinez-Izquierdo J. A. (1991) The sequence of a hydroxyproline-rich glycoprotein gene from Sorghum vulgare. Plant Biol. Mol. 16: $365-367$

77 Chen J and Varner J. E. (1985) Isolation and characterization of cDNA clones for carrot extensin and a proline-rich $33-\mathrm{kDa}$ protein. Proc. Natl. Acad. Sci. USA 82: 4399-4403

78 Kieliszewski M. J and Lamport D. T. A. (1994) Extensin: repetitive motifs, functionnal sites, post-translational codes and phylogeny. Plant J. 5: $157-172$ 